\title{
Dynamical (super)symmetries of monopoles and vortices $\left(^{1}\right)$
}

\author{
P. A. HORVÁTHY \\ Département de Mathématiques, Université \\ Parc de Grandmont, F-37200 TOURS (France)
}

\begin{abstract}
.
The dynamical (super)symmetries for various monopole systems are reviewed. For a Dirac monopole, no smooth Runge-Lenz vector can exist; there is, however, a spectrumgenerating conformal o $(2,1)$ dynamical symmetry that extends into osp $(1 / 1)$ or osp $(1 / 2)$ for spin $1 / 2$ particles. Self-dual 't Hooft-Polyakov-type monopoles admit an su(2/2) dynamical supersymmetry algebra, which allows us to reduce the fluctuation equation to the spin zero case. For large $r$ the system reduces to a Dirac monopole plus an suitable inversesquare potential considered before by McIntosh and Cisneros, and by Zwanziger in the spin 0 case, and to the 'dyon' of D'Hoker and Vinet for spin $1 / 2$. The asymptotic system admits a Kepler-type dynamical symmetry as well as a 'helicity-supersymmetry' analogous to the one Biedenharn found in the relativistic Kepler problem. Similar results hold for the Kaluza-Klein monopole of Gross-Perry-Sorkin. For the magnetic vortex, the $N=2$ supersymmetry of the Pauli Hamiltonian in a static magnetic field in the plane combines with the $o(2) \times o(2,1)$ bosonic symmetry into an $o(2) \times o s p(1 / 2)$ dynamical superalgebra.
\end{abstract}

\section{Introduction.}

The architype of a dynamical symmetry is provided by the Runge-Lenz vector [1] in the Kepler problem,

$$
\mathbf{A}=\frac{1}{2}\{\mathbf{p} \times \mathbf{L}-\mathbf{L} \times \mathbf{p}\}-\mathbf{M} \hat{\mathbf{r}},
$$

where $M$ is the mass of the Sun, the planet's mass is taken to be 1 , and $\mathbf{L}$ denotes the planet's (orbital) angular momentum, $\mathbf{L}=\mathbf{r} \times \mathbf{p}$. The vector $\mathbf{A}$ is directed from the Sun's position towards the perihelion point. Under commutation [Poisson bracket] the RungeLenz vector and the angular momentum close into $o(4)$ for bound (elliptic) motions, into $o(3) \oplus_{s} \mathbf{R}^{3}$ for parabolic motions and into $o(3,1)$ for hyperbolic motions. This makes it possible to calculate the spectrum and the $S$-matrix algebraically.

The Kepler problem also admits an $o(2,1)$ 'spectrum-generating symmetry', which combines with the $o(4) / o(3,1)$ into an irreducible representation of the conformal group $o(4,2)[1]$.

In this Review we examine how similar dynamical symmetries - as well as supersymmetries - arise for various magnetic monopole systems. In the last Chapter, we examine what happens around a magnetic vortex.

$\left({ }^{1}\right)$ Based on a review talk given at the International Symposium on Advanced Topics in Quantum Physics, Shanxi'92. Ed. J.-Q. Liang, M.-L. Wang, S.-N. Qiao, D.C. Su. pp. 283- 293. Science Press, Beijing (1993) [Tours Preprint N. 47/92], and on Tours Preprint N. 60/93 (1993) (unpublished). 
2. The Dirac monopole. [2]

Let us consider a Dirac monopole, whose magnetic field is

$$
\mathbf{B}=g \frac{\mathbf{r}}{r^{3}} .
$$

The conserved angular momentum of a charged, spinless particle is

$$
\mathbf{L}_{0}=\mathbf{r} \times \boldsymbol{\pi}-q \hat{\mathbf{r}}
$$

where $\boldsymbol{\pi}=\mathbf{p}-i q \mathbf{A}_{D}$, rot $\mathbf{A}_{D}=\mathbf{r} / r^{3}, q=e g, e$ being the electric charge. Since

$$
\mathbf{L}_{0} \cdot \hat{\mathbf{r}}=-q
$$

the particle moves classically on a cone of opening angle $\cos \alpha=-q / L_{0}$. There are no bound motions.

The problem of having a conserved Runge-Lenz-type vector naturaly arises, and it has been claimed [3] that the vector A which points from the origin to the closest ('perihelion') point of the trajectory is such a conserved vector, which would generate, with the angular momentum, an $o(3,1)$ dynamical symmetry. This statement is, however, false: a Dirac monopole cannot admit any time-independent, conserved Runge-Lenz-type vector [4]. This can be understood by considering the 'umbrella' transformation of Boulware et al. [5],

$$
\mathbf{r} \mapsto \mathbf{R}=\frac{\mathbf{r}-\hat{\mathbf{L}}_{0}\left(\mathbf{r} \cdot \hat{\mathbf{L}}_{0}\right)}{\sin \alpha}
$$

which rotates the monopole problem into a potential problem: the particle trajectories in the monopole field correspond to those in the plane perpendicular to the angular momentum, $\mathbf{L}_{0}$, in an $-q^{2} / 2 R^{2}$ potential [and makes the $o(2,1)$ symmetry [6] manifest].

The inverse-square potential problem is integrable. Golo's 'Runge-Lenz' vector goes thereby into the vector pointing to the closest point, $\mathbf{R}_{0}$, of the rotated trajectory in the plane perpendicular to $\mathbf{L}_{0}$. This transformation is, however, singular when the motion is radial: when the cone's opening angle closes to zero, the direction of the umbrella transformation becomes undetermined. More precisely, the inverse transformation becomes the familiar Hopf fibering $U(1) \rightarrow S O(3) \rightarrow \mathbf{S}^{2}[4]$.

A spinless particle in the field of a Dirac monopole admits instead an $o(2,1)$ symmetry [6], generated by the 'non-relativistic conformal transformations'

$$
\begin{aligned}
H & = & \frac{1}{2} \boldsymbol{\pi}^{2} & \text { time translations } \\
D & = & t H-\frac{1}{4}\{\boldsymbol{\pi}, \mathbf{r}\} & \text { dilations } \\
K & = & -t^{2} H+2 t D+\frac{1}{2} m r^{2} & \text { expansions }
\end{aligned}
$$

which satisfy the $o(2,1)$ relations

$$
[H, D]=i H, \quad[H, K]=2 i D, \quad[D, K]=i K,
$$


allowing for a derivation of the spectrum from the group theory.

This result can be explained from studying the non-relativistic structure of space-time. A free, non-relativistic particle admits in fact the so-called Schrödinger group as symmetry [7]. This latter is the extension of the Galilei group with dilations and expansions. It is best understood in the five-dimensional framework, where non-relativisic motions are lightlike reductions of null geodesics in a five-dimensional Lorentz manifold [8]. Jackiw's $o(2,1)$ is just the residual symmetry left over from the Schrödinger group after adding a Dirac monopole.

The only potential which is consistent with the conformal algebra $(2.5)$ is $\lambda^{2} / r^{2}$ : for an arbitrary $\lambda$, the Hamiltonian

$$
\frac{1}{2}\left[\boldsymbol{\pi}^{2}+\frac{\lambda^{2}}{r^{2}}\right]
$$

is $o(2,1)$ symmetric. Adding a Coulomb term would break this symmetry. However, as first noticed by Zwanziger, and by McIntosh and Cisneros (MCZ) [9], a slightly different system does have a Kepler-type dynamical symmetry. It consists of Dirac monopole plus a fine-tuned inverse-square potential plus a Coulomb term,

$$
H_{M C Z}=\frac{1}{2}\left(\pi^{2}+\frac{\alpha}{r}+\frac{q^{2}}{r^{2}}\right)
$$

which admits a conserved Runge-Lenz vector, namely

$$
\mathbf{A}_{0}=\frac{1}{2}\left\{\boldsymbol{\pi} \times \mathbf{L}_{0}-\mathbf{L}_{0} \times \boldsymbol{\pi}\right\}-q^{2} \hat{\mathbf{r}} .
$$

This is understood by noting that, when applying the 'umbrella-transformation' (2.4) the $q^{2} / 2 r^{2}$ potentials cancel and we are left with an effective Kepler problem. The $o(4) / o(3,1)$ dynamical symmetry generated by $\mathbf{L}_{0}$ and $\mathbf{A}_{0}$ can be used to determine the spectrum and the scattering matrix [9], respectively. It extends into $o(4,2)$, but in another representation as for Kepler [1,10].

Jackiw's result was generalized [11] to a spin $\frac{1}{2}$ particle with gyromagnetic ratio 2 , described by the two-component Pauli Hamiltonian

$$
H_{P}=\frac{1}{2}\left(\boldsymbol{\pi}^{2}-q \frac{\boldsymbol{\sigma} \cdot \hat{\mathbf{r}}}{r^{2}}\right) .
$$

This system has not only the bosonic $o(2,1)$ with $D, K$ in $(2.5)$ as for spin 0 , but also two conserved supercharges, namely

$$
Q=\frac{1}{\sqrt{2}} \boldsymbol{\sigma} \cdot \boldsymbol{\pi} \quad \text { and } \quad S=\frac{1}{\sqrt{2}} \boldsymbol{\sigma} \cdot \mathbf{r}-t Q,
$$

which close with the bosonic generators into an $\operatorname{osp}(1 / 1)$ superalgebra, i.e. (2.6), supplemented by

$$
\begin{aligned}
& {[Q, D]=\frac{i}{2} Q, \quad[K, D]=i S, \quad[S, H]=i Q, \quad[S, D]=-\frac{i}{2} S,} \\
& {[Q, H]=0, \quad[S, K]=0,} \\
& \{Q, Q\}=2 H \quad\{Q, S\}=-2 D, \quad\{S, S\}=2 K .
\end{aligned}
$$


The $\operatorname{osp}(1,1)$ symmetry, which allows to derive the spectrum algebraically, can be seen to be the residual superalgebra of the 'super-Schrödinger algebra', obtained from adding the (fermionic) 'helicity operator' $Q$ in (2.11) to the Schrödinger group [12].

\section{Supersymmetric quantum mechanics.}

D'Hoker and Vinet [13,14] have further generalized the problem. To explain their results, let us consider a four-dimensional, euclidean space and choose the representation

$$
\gamma^{k}=\left(\begin{array}{cc}
0 & \sigma^{k} \\
\sigma^{k} & 0
\end{array}\right), \quad \gamma^{4}=\left(\begin{array}{cc}
0 & -i 1_{2} \\
i 1_{2} & 0
\end{array}\right), \quad \gamma^{5}=\left(\begin{array}{cc}
1_{2} & 0 \\
0 & -1_{2}
\end{array}\right)
$$

for the Dirac matrices. Let $A_{\mu}$ denote a gauge field. The four dimensional Dirac operator,

$$
\not \varnothing \equiv \gamma^{\mu}\left(\partial_{\mu}-i A_{\mu}\right) \equiv\left(\begin{array}{cc}
Q^{\dagger} \\
Q
\end{array}\right)
$$

is, as in any even dimensions chiral-supersymmetric. This means that the square of $\not D$,

$$
\not D^{2}=\left(\begin{array}{ll}
H_{1} & \\
& H_{0}
\end{array}\right)
$$

is a supersymmetric hamiltonian. Its \pm 1 chirality sectors (eigensectors of $\gamma^{5}$ ) are related by the unitary transformations

$$
U=Q \frac{1}{\sqrt{H_{1}}} \quad \text { and } \quad U^{-1} \equiv U^{\dagger}=\frac{1}{\sqrt{H_{1}}} Q^{\dagger}
$$

which intertwine $H_{1}=Q^{\dagger} Q$ and $H_{0}=Q Q^{\dagger}, H_{1}=U^{\dagger} H_{0} U$. If $\Psi_{0}$ is an $H_{0}$-eigenfunction with eigenvalue $E>0$, then

$$
\left(\begin{array}{c}
U^{\dagger} \Psi_{0} \\
\pm \Psi_{0}
\end{array}\right)
$$

is a $\not \not$-eigenfunction with eigenvalues $\pm \sqrt{E}$. Zero-energy ground states may arise; the difference of their multiplicities in the two sectors, called the Atiyah-Singer index, is calculated by topological formulae.

Furthermore, if $A_{0}$ is conserved for $H_{0},\left[A_{0}, H_{0}\right]=0$, then

$$
A_{1}=U^{\dagger} A_{0} U
$$

is conserved for $H_{1},\left[A_{1}, H_{1}\right]=0$.

Let us first apply these framework to the gauge field

$$
\mathbf{A}=q \mathbf{A}_{D}, \quad A_{4}=\frac{\lambda}{r},
$$


where $\lambda$ is an arbitrary real constant. This gauge field represents a Dirac monopole plus a Coulomb potential in the fourth (euclidean) direction. Assuming that nothing depends on the fourth direction, $\partial_{4}()=$.0 , the associated Dirac operator becomes

$$
\begin{aligned}
\frac{1}{\sqrt{2}} \not \supset= & \frac{1}{\sqrt{2}}\left(\begin{array}{c}
Q^{\dagger} \\
Q
\end{array}\right)=\frac{1}{\sqrt{2}}\left(\gamma^{i} \pi_{i}+\gamma^{4} \frac{\lambda}{r}\right)= \\
& \frac{1}{\sqrt{2}}\left(\begin{array}{l}
\boldsymbol{\sigma} \cdot \boldsymbol{\pi}-i \frac{\lambda}{r} \\
\boldsymbol{\sigma} . \boldsymbol{\pi}+i \frac{\lambda}{r}
\end{array}\right) .
\end{aligned}
$$

Its square is the four-component Hamiltonian

$$
H=\frac{1}{2}\left\{\boldsymbol{\pi}^{2}-q \frac{\boldsymbol{\sigma} \cdot \hat{\mathbf{r}}}{r^{2}}+\frac{\lambda^{2}}{r^{2}}-\lambda \gamma^{5} \frac{\boldsymbol{\sigma} \cdot \hat{\mathbf{r}}}{r^{2}}\right\} .
$$

The Hamiltonian (3.9) is block-diagonal, and the \pm 1 chirality components only differ in the sign of $\lambda$. They describe two uncoupled spin $\frac{1}{2}$ particles with anomalous gyromagnetic ratios.

Interestingly, the Hamiltonian (3.9) is a perfect square in two different ways:

$$
Q_{1}=\frac{1}{\sqrt{2}} \gamma^{5}\left(\gamma^{i} \pi_{i}+\gamma^{4} \frac{\lambda}{r}\right) \quad \text { and } \quad Q_{2}=-i \gamma^{5} Q_{1}
$$

both satisfy $\left\{Q_{a}, Q_{b}\right\}=\delta_{a b} H$, and are hence conserved. They mix with the bosonic $o(2,1)$ symmetry, yielding two more supercharges, namely

$$
S_{1}=-t Q+\frac{1}{\sqrt{2}} \gamma^{5} \gamma^{i} r_{i} \quad \text { and } \quad S_{2}=-i \gamma^{5} S_{1}
$$

which satisfy $\left\{S_{a}, S_{b}\right\}=2 \delta_{a b} K$. Finally,

$$
\left\{Q_{a}, S_{b}\right\}=-2 \delta_{a b} D+2 \epsilon_{a b} Y
$$

where $Y$ is the parity operator

$$
Y=\frac{1}{2} \gamma^{5}\left(\boldsymbol{\sigma} \cdot \ell+\frac{3}{2}-\lambda \gamma^{5} \frac{\boldsymbol{\sigma} \cdot \hat{\mathbf{r}}}{r}\right), \quad \text { where } \quad \ell=\mathbf{r} \times \boldsymbol{\pi} .
$$

The four bosonic operators $H, D, K, Y$ close with the fermionic operators $Q_{a}, S_{a}(\mathrm{a}=1$, 2 ) into the superalgebra $\operatorname{osp}(1 / 2)$.

Since the field (3.7) is manifestly spherically symmetric, the total angular momentum,

$$
\mathbf{J}=\mathbf{L}_{0}+\frac{1}{2} \boldsymbol{\sigma}
$$

is also conserved. 
For the special value $q= \pm \lambda$, the Pauli term drops out from one of the sectors while the gyromagnetic ratio becomes 4 in the other. Eq. (3.9) reduces hence to

$$
H=\left(\begin{array}{cc}
H_{1} & \\
& H_{0}
\end{array}\right)=\frac{1}{2}\left\{\boldsymbol{\pi}^{2}+\frac{\lambda^{2}}{r^{2}}+\frac{2 q}{r^{2}}\left(\begin{array}{c}
\boldsymbol{\sigma} \cdot \hat{\mathbf{r}} \\
0
\end{array}\right)\right\} .
$$

Being spin-independent, the lower Hamiltonian clearly admits

$$
\mathbf{S}_{0}=\frac{1}{2} \boldsymbol{\sigma}
$$

as symmetry. But supersymmetry implies that its partner Hamiltonian has also a 'spin' symmetry,

$$
\mathbf{S}_{1}=U^{\dagger} \mathbf{S}_{0} U
$$

commutes with $H_{1} \cdot \mathbf{S}_{0}$ and $\mathbf{L}_{0}=\mathbf{J}-\mathbf{S}_{0}$ are hence both conserved for $H_{0}$. Thus, $\mathbf{S}_{1}$ and

$$
\mathbf{L}_{1}=U^{\dagger} \mathbf{L}_{0} U=\mathbf{J}-\mathbf{S}_{1}=\mathbf{L}_{0}+\frac{1}{2} \boldsymbol{\sigma}-\mathbf{S}_{1}
$$

are both conserved for $H_{1}$ The combined system $\left(\begin{array}{cc}H_{1} & \\ & H_{0}\end{array}\right)$ has therefore two conserved 'angular momenta', namely

$$
\mathbf{S}=\left(\begin{array}{cc}
\mathbf{S}_{1} & \\
& \mathbf{S}_{0}
\end{array}\right) \quad \text { and } \quad \mathbf{L}=\left(\begin{array}{cc}
\mathbf{L}_{1} & \\
& \mathbf{L}_{0}
\end{array}\right) .
$$

The action of the supercharges extends the $o(3)_{\text {spin }}$ algebra into $u(2 / 2)$. Let us indeed define the vector supercharges

$$
\mathbf{Q}_{\alpha}=2 i\left[\mathbf{S}_{0}, Q_{\alpha}\right] \quad(\alpha=1,2),
$$

i.e.

$$
\mathbf{Q}_{1}=\left(\begin{array}{ll}
2 i \mathbf{S}_{0} Q & -2 i Q^{\dagger} \mathbf{S}_{0}
\end{array}\right), \quad \mathbf{Q}_{2}=\left(\begin{array}{c}
-2 Q^{\dagger} \mathbf{S}_{0} \\
-2 \mathbf{S}_{0} Q
\end{array}\right)
$$

All these operators commute with the Hamiltonian $H$. One has furthermore

$$
\left[\gamma^{5}, \text { bosonic }\right]=0 \quad\left\{\gamma^{5}, \text { fermionic }\right\}=0 .
$$

To summarize, the bosonic operators $\mathbf{S}_{0}, \mathbf{S}_{1}, \gamma^{5}, H$ and the fermionic operators $\mathbf{Q}_{a}, Q_{a}$ satisfy the (anti)commutation relations

$$
\begin{aligned}
& {\left[S_{0}^{i}, S_{0}^{j}\right]=i \epsilon_{i j k} S_{0}^{k},} \\
& {\left[S_{1}^{i}, S_{1}^{j}\right]=i \epsilon_{i j k} S_{1}^{k},} \\
& {\left[S_{0}^{i}, S_{1}^{j}\right]=0} \\
& {\left[\gamma_{5}, S_{0}^{i}\right]=\left[\gamma_{5}, S_{1}^{i}\right]=0}
\end{aligned}
$$

bosonic sector 


$$
\left.\begin{array}{l}
{\left[\gamma^{5}, Q_{a}\right]=2 i \epsilon_{a b} Q_{b} \quad(a, b=1,2)} \\
{\left[\gamma^{5}, Q_{a}^{k}\right]=2 i \epsilon_{a b} Q_{b}^{k} \quad(a, b=1,2, k=1,2,3)} \\
{\left[S_{0}^{i}, Q_{a}^{j}\right]=\frac{i}{2}\left(\delta_{i j} Q_{a}+\epsilon_{i j k} Q_{a}^{k}\right),} \\
{\left[S_{0}^{i}, Q_{a}^{j}\right]=-\frac{i}{2} Q_{a}^{i},} \\
{\left[S_{1}^{i}, Q_{a}^{j}\right]=-\frac{i}{2}\left(\delta_{i j} Q_{a}-\epsilon_{i j k} Q_{a}^{k}\right),} \\
{\left[S_{1}^{i}, Q_{a}\right]=\frac{i}{2} Q_{a}^{i}}
\end{array}\right\} \begin{gathered}
\text { action of } \\
\text { bosonic operators } \\
\text { on fermionic sector }
\end{gathered}
$$

fermionic sector

$$
\left.\begin{array}{l}
\left\{Q_{a}, Q_{a}\right\}=2 \delta_{a b} H, \\
\left\{Q_{a}, Q_{b}^{i}\right\}=-4 H \epsilon_{a b}\left(S_{0}^{i}+S_{1}^{i}\right), \\
\left\{Q_{a}^{i}, Q_{b}^{j}\right\}=2 H \delta_{i j} \delta_{a b}-4 H \epsilon_{i j k} \epsilon_{a b}\left(S_{1}^{i}-S_{1}^{i}\right),
\end{array}\right\}
$$

i.e. close into the $s u(2 / 2)$ SUSY algebra $[14,17,18]$. The $\operatorname{osp}(2,1)$ found before mixes with the $o(3)_{\text {rotations }}$ and the $u(2 / 2)_{\text {spin }}$ to yield a supersymmetric version of $o(4,2)$. Its precise structure has not yet been determined.

\section{Self-dual 't Hooft-Polyakov monopoles.}

The Dirac monopole was generalized by 't Hooft and Polyakov in non-Abelian gauge theory [2]. It is a static, purely magnetic $\left(\partial_{0}=0\right)$, everywhere-regular, finite-energy solution to the $S U(2)$ Yang-Mills Higgs equations associated to the energy functional

$$
E=\int d^{3} x\left\{\frac{1}{4} \operatorname{Tr}\left(F_{i j} F^{i j}\right)+\frac{1}{2} \operatorname{Tr}\left(D_{j} \Phi D^{j} \Phi\right)+\frac{\lambda}{4}\left[1-\operatorname{Tr}\left(\Phi^{2}\right)\right]^{2}\right\},
$$

where $F_{i j}=\partial_{i} A_{j}-\partial_{j} A_{i}+\left[A_{i}, A_{j}\right]$ and $D_{j} \Phi=\partial_{j} \Phi+\left[A_{j}, \Phi\right]$.

Finite-energy requires $|\Phi| \simeq 1$ for large $r$, so that the asymptotic values of the Higgs field define a mapping from the 'sphere at infinity' $\mathbf{S}^{2}$ into the 'vacuum manifold' $\mathcal{M}=$ $|\Phi|=1$. $\mathcal{M}$ is again a two-sphere, so it provides us with the integer

$$
n=[\Phi] \in \pi_{2}\left(\mathbf{S}^{2}\right) \simeq \mathbf{Z}
$$

called the topological charge.

For non-vanishing Higgs potential (i.e. $\lambda \neq 0$ ), the sytem has the same $o(2,1)$ bosonic symmetry as the Dirac monopole.

In the 'Prasad-Sommerfield limit' of vanishing $\lambda$ the situation is different. The secondorder field equations associated to (4.1) are solved by the 'self-duality' or 'Bogomolny' equations

$$
\mathbf{B}=\mathbf{D} \Phi \quad \text { where } \quad B_{i}=\frac{1}{2} \epsilon_{i j k} F^{j k}
$$


For $n=1$ for exemple, Prasad and Somerfield found the solution

$$
A_{j}^{a}=\epsilon_{a j k}\left(1-\frac{r}{\sinh r}\right) \frac{x^{k}}{r^{2}}, \quad \Phi^{a}=-\left(\operatorname{coth} r-\frac{1}{r}\right) \frac{x^{a}}{r} .
$$

Setting $A_{4}=\Phi$ and requiring $\partial_{4}=0$, a PS monopole can also be viewed as a self-dual Yang-Mills field in four euclidean dimensions.

Let us now consider a massless Dirac particle in a BPS background, described by the four dimensional Dirac operator

$$
\not D=\left(\begin{array}{ll}
Q^{\dagger} \\
Q &
\end{array}\right)=\left(\begin{array}{cc}
\boldsymbol{\sigma} \cdot \boldsymbol{\pi}+i \Phi & \boldsymbol{\sigma}-i \Phi
\end{array}\right) .
$$

As explained in Section 3, $\not D$ is chiral-supersymmetric. Now, owing to

$$
Q Q^{\dagger}=\pi^{2}+\Phi^{2}+\boldsymbol{\sigma} \cdot(\mathbf{B}-\mathbf{D} \Phi), \quad Q^{\dagger} Q=\boldsymbol{\pi}^{2}+\Phi^{2}+\boldsymbol{\sigma} \cdot(\mathbf{B}+\mathbf{D} \Phi)
$$

the spin drops out in the self-dual sector, while we get a factor 2 in the other one: $H_{0}$ describes two spin 0 particle (or a spin $\frac{1}{2}$ particle with gyromagnetic ratio 0 ), while $H_{1}$ describes a particle with anomalous gyromagnetic ratio 4 . This is why the fluctuation equation in the BPS background can be reduced to the study of the spin 0 system $[15,16]$.

The spin operator $\mathbf{S}_{0}=\boldsymbol{\sigma} / 2$ is trivially conserved for $H_{0}$. Its superpartner,

$$
\mathbf{S}_{1}=U^{\dagger} \mathbf{S}_{0} U=\frac{1}{H_{1}}\left\{\frac{1}{2}\left[\boldsymbol{\pi}^{2}-\Phi^{2}\right] \boldsymbol{\sigma}+\Phi(\boldsymbol{\pi} \times \boldsymbol{\sigma})-(\boldsymbol{\sigma} . \boldsymbol{\pi}) \boldsymbol{\pi}\right\}
$$

is therefore conserved for $H_{1}$.

Zero-energy ground states only arise for $H_{1}$ (but not for $H_{0}$ ) as solutions of $Q \Psi=0$. The multiplicity of these states (the Atiyah-Singer index) was found to be $2 n$, twice the topological charge [16].

Since BPS monopoles with topological charge $n \geq 2$ are not spherically symmetric, for a general BPS monopole this is the end of the story. For the $n=1$ the BPS solution above, however, we also have spherical symmetry and hence the total angular momentum, $\mathbf{J}=\mathbf{L}_{0}+\frac{1}{2} \boldsymbol{\sigma}$, is conserved. The same argument as in Section 3 shows that

$$
\mathbf{L}_{0}=\mathbf{J}-\mathbf{S}_{0} \quad \text { and } \quad \mathbf{L}_{1}=U \mathbf{L}_{0} U^{\dagger}=\mathbf{J}-\mathbf{S}_{1}
$$

cf. (3.17) are conserved for $H_{0}$ and $H_{1}$ respectively; the commuting operators $\mathbf{L}$ and $\mathbf{S}$ in Eq. (3.18) generate $o(3)_{\text {rotations }} \oplus o(3)_{\text {spin }}$, and the spin part is extended into $u(2 / 2)$ as in Eq. (3.21).

\section{Dyons.}

For large $r$, the systems become even more symmetric. The BPS monopole becomes an imbedded Dirac monopole with an additional long-range scalar field $\Phi \sim 1-1 / r$. For eigenstates of the electric charge operator $Q_{e m}=\hat{\Phi}$ the $S U(2)$-covariant derivative 
reduces to the electromagnetic covariant derivative with coupling constant equal $q$, the electric charge. Thus,

$$
\begin{aligned}
& H_{0} \longrightarrow H_{M C Z}=\pi^{2}+q^{2}\left(1-\frac{1}{r}\right)^{2} \\
& H_{1} \longrightarrow H_{D}=\pi^{2}+q^{2}\left(1-\frac{1}{r}\right)^{2}+2 q \frac{\boldsymbol{\sigma} \cdot \mathbf{r}}{r^{3}}
\end{aligned}
$$

Remarkably, the large- $r$ limit of $H_{0}$ is precisely the $H_{M C Z}$, the McIntosh - Cisneros Zwanziger Hamiltonian (2.8) (times the unit $2 \times 2$ matrix), while its partner $H_{1}$ becomes the 'dyon' Hamiltonian $H_{D}$ of D'Hoker and Vinet $[17,18]$. Supersymmetry then converts the Runge-Lenz vector $\mathbf{A}_{0}$ of MCZ into a spin-dependent Runge-Lenz vector,

$$
\mathbf{A}_{1}=\underbrace{\left\{\frac{1}{2}\left(\boldsymbol{\pi} \times \mathbf{L}_{0}-\mathbf{L}_{0} \times \boldsymbol{\pi}\right)-q^{2} \hat{\mathbf{r}}\right\}}_{\mathbf{A}_{0}}+\boldsymbol{\pi} \times \boldsymbol{\sigma}+\frac{q}{r} \boldsymbol{\sigma}-q \frac{\mathbf{r} \cdot \boldsymbol{\sigma}}{r^{3}} \mathbf{r}-\frac{q}{2} \boldsymbol{\sigma}
$$

which is conserved for $H_{D}$. For the asymptotic system

$$
\left(\begin{array}{cc}
H_{D} & \\
& H_{M C Z}
\end{array}\right)
$$

the bosonic symmetry algebra $o(3)_{\text {rotations }} \oplus o(3)_{\text {spin }}$ extends therefore into

$$
o(4) \oplus o(3)_{\operatorname{spin}}
$$

for bound motions (and into $o(4) \oplus o(3)_{\text {spin }} / o(4) \oplus o(3)_{\text {spin }}$ for scattered motions) $\left(^{1}\right)$, generated by

$$
\mathbf{A}=\left(\begin{array}{ll}
\mathbf{A}_{1} & \\
& \mathbf{A}_{0}
\end{array}\right)
$$

and by $\mathbf{L}$ and $\mathbf{S}$ in Eq. (3.17), to which is added the supersymmetry algebra $u(2 / 2)$ in Eq. $(3.21)$.

The dynamical symmetry (5.4) makes it possible to find the spectrum $[14,18,19]$,

$$
E=q^{2}\left(1-\frac{q^{2}}{p^{2}}\right), \quad p=\begin{gathered}
q, q+1, \ldots \\
q+1, \ldots
\end{gathered} \text { for } \quad \begin{aligned}
& H_{1} \\
& H_{0}
\end{aligned} .
$$

Chiral SUSY means that the spectra of $H_{0}$ and of $H_{1}$ are identical up to zero-energy ground-states. Closer inspection shows, however, even more symmetry, namely a two-fold degeneracy.

$\left({ }^{1}\right)$ It is likely that this symmetry is further extended to $o(4,2) \oplus o(3)_{\text {spin }}$. 
Let us focus our attention to a fixed $j=$ const. sector. The pattern is reminiscent of a supersymmetric system except that the ground state energy is nonzero.

Generalizing Biedenharn's approach to the relativistic Kepler problem [20], we can exhibit another conserved operator, namely

$$
\mathcal{R}=\left(\begin{array}{ll} 
& R^{\dagger}
\end{array}\right)=\left(\begin{array}{cc}
i \boldsymbol{\sigma} \cdot \boldsymbol{\pi}+\frac{q}{r}+(\boldsymbol{\sigma} \cdot \hat{\mathbf{r}}) \frac{q^{2}}{x} \\
i \boldsymbol{\sigma} \cdot \boldsymbol{\pi}-\frac{q}{r}+(\boldsymbol{\sigma} \cdot \hat{\mathbf{r}}) \frac{q^{2}}{y} &
\end{array}\right)
$$

we call 'dyon helicity' operator [19]. Here

$$
\begin{aligned}
& x=\boldsymbol{\sigma} \cdot \ell+1-q \hat{\boldsymbol{\sigma}} \cdot \hat{\mathbf{r}} \\
& y=\boldsymbol{\sigma} \cdot \ell+1+q \hat{\boldsymbol{\sigma}} \cdot \hat{\mathbf{r}}
\end{aligned} \quad \text { is conserved for } \quad \begin{gathered}
H_{0} \\
H_{1}
\end{gathered} \quad(\ell=\mathbf{r} \times \mathbf{p}) .
$$

$x$ and $y$ both have the eigenvalues $\pm(j+1 / 2)[18,19]$. They are just the components of the Biedenharn-Temple operator

$$
\Gamma=-\left(\boldsymbol{\sigma} \cdot \ell+q \gamma^{5} \boldsymbol{\sigma} \cdot \hat{r}\right)=\left(\begin{array}{cc}
-y & \\
& -x
\end{array}\right) .
$$

Since the dyon helicity operator $\mathcal{R}$ satisfies

$$
\mathcal{R}^{2}=\not D^{2}-E_{0}^{(j)}
$$

Subtracting the ground-state energy $E_{0}^{(j)}$,

$$
\not D^{2}-E_{0}^{(j)}=\left(\begin{array}{ll}
H_{1}-q^{2}+\frac{q^{4}}{(j+1 / 2)^{2}} & \\
& H_{0}-q^{2}+\frac{q^{4}}{(j+1 / 2)^{2}}
\end{array}\right)
$$

becomes hence a supersymmetric, with $\mathcal{R}$ as square-root. The new supersymmetry-sectors are the \pm 1 eigensectors of the normalized Biedenharn-Temple operator $\Gamma /|\Gamma|$.

The dyon helicity operator has the nice property that it respects the angular decomposition. Explicit eigenfunctions are constructed in Ref. [19].

\section{Particle in the Wu-Yang monopole field.}

The MCZ system has yet another symmetric generalizations. Rather then considering spin $\frac{1}{2}$ particles, with vanishing isospin, we can also study spin 0 particles with isospin, moving in a self-dual Wu-Yang [21] monopole field. This latter is obtained by imbedding the Dirac monopole into $S U(2)$ gauge theory and adding a suitable 'hedgehog' scalar field,

$$
\mathbf{A}=\frac{i}{2 r} \boldsymbol{\sigma} \times \hat{\mathbf{r}}, \quad \Phi=\frac{i}{2}\left(1-\frac{1}{r}\right) \boldsymbol{\sigma} \cdot \hat{\mathbf{r}} .
$$


The electric charge is defined [2] as the eigenvalue of

$$
Q_{e m}=\hat{\Phi}=\boldsymbol{\sigma} . \hat{\mathbf{r}} \text {. }
$$

The Hamiltonian is hence

$$
H_{W Y}=\frac{1}{2}\left(-i \nabla-Q_{e m} \mathbf{A}_{D}\right)^{2}+\frac{Q_{e m}^{2}}{2}\left(1-\frac{1}{r}\right)^{2} .
$$

Since on the $Q_{e m}= \pm q$, eigensectors $H_{W Y}$ reduces to the MCZ Hamiltonian, such a particle admits the conserved Runge-Lenz vector [22]

$$
\begin{aligned}
\mathbf{A}= & \frac{1}{2}\{\boldsymbol{\pi} \times \mathbf{J}-\mathbf{J} \times \boldsymbol{\pi}\}-q^{2} \hat{\mathbf{r}} \\
& +\frac{1}{2 r}\left\{q \boldsymbol{\sigma}-q(\boldsymbol{\sigma} \cdot \hat{\mathbf{r}}) \hat{\mathbf{r}}--\frac{i}{2} \boldsymbol{\sigma} \times \hat{\mathbf{r}}-r \boldsymbol{\sigma} \times \boldsymbol{\pi}+(\boldsymbol{\sigma} \cdot \ell+1) \hat{\mathbf{r}}\right\} .
\end{aligned}
$$

A variation of the model can be obtained by considering 'nucleon-type' particles [13, 23 ], whose electric charge operator is

$$
Q_{e m}^{\prime}=Q_{e m}-\frac{1}{2} \boldsymbol{\sigma} \cdot \hat{\mathbf{r}}
$$

The associated Hamiltonian is only slightly different from yet another one studied by D'Hoker and Vinet [13], namely

$$
H_{N}=\frac{1}{2}\left\{\left(-i \nabla-\left(Q_{e m}-\frac{1}{2} \boldsymbol{\sigma} \cdot \hat{\mathbf{r}}\right) \mathbf{A}_{D}\right)^{2}+\frac{q^{2}+1 / 4-\boldsymbol{\sigma} \cdot \hat{\mathbf{r}} / 2}{r^{2}}+\frac{\alpha}{r}\right\} .
$$

This admits again a conserved Runge-Lenz vector, namely [23]

$$
\begin{aligned}
\mathbf{A}= & \frac{1}{2}\left\{\boldsymbol{\pi} \times \mathbf{L}_{0}-\mathbf{L}_{0} \times \boldsymbol{\pi}\right\}-q^{2} \hat{\mathbf{r}} \\
& +\frac{1}{2 r}\left\{q \boldsymbol{\sigma}-q(\boldsymbol{\sigma} \cdot \hat{\mathbf{r}}) \hat{\mathbf{r}}--\frac{i}{2} \boldsymbol{\sigma} \times \hat{\mathbf{r}}-r \boldsymbol{\sigma} \times \boldsymbol{\pi}+(\boldsymbol{\sigma} \cdot \ell+1) \hat{\mathbf{r}}\right\} .
\end{aligned}
$$

D'Hoker and Vinet have also proved that $H_{N}$ is actually a partner Hamiltonian of a supersymmetric system, namely of

$$
\left(\begin{array}{ll}
H_{N} & \\
& H_{D}
\end{array}\right)
$$

\section{The Kaluza-Klein monopole.}

The Kaluza-Klein monopole [24] is obtained by imbedding the Taub-NUT gravitational instanton as a static soliton in Kaluza-Klein theory. This latter is described by the 4-metric

$$
\begin{aligned}
& V\left\{d r^{2}+r^{2}\left(d \theta^{2}+\sin ^{2} \theta d \phi^{2}\right)\right\}+\frac{1}{V}\{d \psi+4 m \cos \theta d \phi\}^{2} \\
& \text { where } \quad V=1+\frac{4 m}{r}
\end{aligned}
$$


The 'vertical' variable $\psi$ describes a internal circle. The apparent singularity at $r=0$ is unphysical if $\psi$ is periodic with period $16 \pi m$. In the usual context, the Taub-NUT parameter, $m$ is positive. We shall, however, also consider $m<0$. Such a situation arises, e.g. in the long-range scattering of self-dual $S U(2)$ monopoles [25].

$\partial_{\psi}$ is a Killing vector, and the associated conserved quantity, $q$ is quantized in halfintegers. It is identified with the electric charge.

The curved-space gamma - matrices $\hat{\gamma}^{A}$ and the spin connection $\Gamma^{A}$ in the KK monopole background are found to be

$$
\hat{\gamma}^{j}=\left(\begin{array}{cc}
0 & -\frac{i}{\sqrt{V}} \boldsymbol{\sigma} \\
\frac{i}{\sqrt{V}} \boldsymbol{\sigma} & 0
\end{array}\right) \quad \hat{\gamma}^{4}=\left(\begin{array}{cc}
0 & \sqrt{V}+\frac{i}{\sqrt{V}} \boldsymbol{\sigma} \cdot \mathbf{A} \\
\sqrt{V}-\frac{i}{\sqrt{V}} \boldsymbol{\sigma} \cdot \mathbf{A} & 0
\end{array}\right)
$$

and

$$
\Gamma^{i}=\left(\begin{array}{cc}
-\frac{1}{2 V^{2}}(\boldsymbol{\sigma} \cdot \mathbf{B}) A^{i}+\frac{1}{2 V}(\mathbf{B} \times \boldsymbol{\sigma})^{i} & 0 \\
0 & 0
\end{array}\right), \quad \Gamma_{4}=\left(\begin{array}{cc}
-\frac{1}{2 V^{2}} \mathbf{B} \cdot \boldsymbol{\sigma} & 0 \\
0 & 0
\end{array}\right)
$$

Requiring that all fields be equivariant with respect to the vertical action $\psi \mapsto \psi+\alpha$ i.e. have the form, $e^{i q \psi} \Psi$, The Dirac operator becomes [26]

$$
\not D=\left(\begin{array}{cc}
0 & Q^{\dagger} \\
Q & 0
\end{array}\right)=\left(\begin{array}{cc}
0 & \frac{1}{\sqrt{V}} \boldsymbol{\sigma} \cdot \boldsymbol{\pi}-i \frac{q}{4 m} \sqrt{V} \\
\frac{1}{V} \boldsymbol{\sigma} . \boldsymbol{\pi} \sqrt{V}+i \frac{q}{4 m} \sqrt{V} & 0
\end{array}\right),
$$

where $\boldsymbol{\pi}=-i \nabla-(q / 4 m) \mathbf{A}, \mathbf{A}$ being the vectorpotential of a Dirac monopole of unit strength. (It is easy to check that $Q$ and $Q^{\dagger}$ are each other's adjoint with respect to the Taub-NUT volume element $V d^{4} x$, as they have to be). Using the self-duality property

$$
\nabla V=\mathbf{B}
$$

the square of $\not D$ is readily found to be

$$
\left(\begin{array}{rr}
H_{0}+\frac{1}{V}\left[-\frac{q}{r^{2} V} \boldsymbol{\sigma} \cdot \hat{\mathbf{r}}+4 m \frac{\boldsymbol{\sigma} \cdot \mathbf{L}_{0}}{r^{3} V}+\frac{12 m^{2}}{r^{4} V^{2}}\right] & \\
& \frac{1}{V}\left[\boldsymbol{\pi}^{2}+\left(\frac{q}{4 m}\right)^{2} V^{2}\right]
\end{array}\right),
$$

$\mathbf{L}_{0}$ being the spin-0 'monopole' orbital angular momentum, $\mathbf{L}_{0}=\mathbf{r} \times \boldsymbol{\pi}-q \hat{\mathbf{r}}$ in Eq. (2.2). ( $\mathbf{L}_{0}$ is conserved only for $H_{0}$ but not for $H_{1}$ ). The partner hamiltonians $H_{1}$ and $H_{0}$ differ hence in a complicated expression, and it is not at all obvious that they will have the same spectra. Chiral SUSY implies however that this is nevertheless true.

Let us first focus our attention to the $\gamma^{5}=-1$ sector. Observe now that the spin dependence has again dropped out, so it actually describes two, uncoupled, spin 0 particles. $H_{0}$ is in fact the same as the Hamiltonian for a spin 0 particle in the KK field [4] (times the unit matrix). 
Because the spin is uncoupled, the system again has two angular momenta, namely orbital angular momenta and the spin vectors,

$$
\mathbf{L}_{0} \quad \mathbf{L}_{1}=U^{\dagger} \mathbf{L}_{0} U, \quad \text { and } \quad \mathbf{S}_{0}=\frac{\boldsymbol{\sigma}}{2} \quad \mathbf{S}_{1}=U^{\dagger} \mathbf{S}_{0} U
$$

cf. (3.17-18). $H_{0}$ admits [25] a Runge-Lenz vector,

$$
\mathbf{A}_{0}=\frac{1}{2}\{\boldsymbol{\pi} \times \mathbf{L}-\mathbf{L} \times \boldsymbol{\pi}\}-4 m \hat{\mathbf{r}}\left(H_{0}-\left(\frac{q}{4 m}\right)^{2}\right)
$$

The vector operators $\mathbf{L}_{\mathbf{0}}$ and $\mathbf{K}_{0}$ generate an $o(3,1)$ dynamical symmetry for scattered motions and $o(4)$ for bound motions. Its superpartner, $\mathbf{A}_{1}=U^{\dagger} \mathbf{A}_{0} U$ [cf. (5.5)], generates an analogous dynamical symmetry group for $H_{1}[26]$.

\section{Supersymmetry of the magnetic vortex}

The three-dimensional (super)symmetries studied above become even larger in the plane $[27,28]$, namely for a magnetic vortex (an idealization for the Aharonov-Bohm experiment). Firstly, the $o(2,1)$ symmetry (2.5) is still present; on the other hand, the $N=2$ supersymmetry of the Pauli Hamiltonian of a spin- $\frac{1}{2}$ particle, present for any magnetic field in the plane [29], combines, for a magnetic vortex, with Jackiw's $o(2) \times o(2,1)$ into an $o(2) \times \operatorname{osp}(1 / 2)$ superalgebra $\left({ }^{2}\right)$. This curious supersymmetry is realized with two (rather then four)-component objects, and is only possible in two spatial dimensions [30]. It arises owing to the existence of two "scalar products" in the plane, namely the ordinary (symmetric) scalar product, and the (antisymmetric) vector product $\left({ }^{3}\right)$.

In detail, let us first consider a spin- $\frac{1}{2}$ particle in an arbitrary static magnetic field $\mathbf{B}=(0,0, B), B=B(x, y)$. Dropping the irrelevant $z$ variable, we work in the plane. Then our model is described by the Pauli Hamiltonian

$$
H=\frac{1}{2 m}\left[\pi^{2}-e B \sigma_{3}\right]
$$

where $B=\operatorname{rot} \mathbf{A}\left(\equiv \epsilon^{i j} \partial_{i} A_{j}\right)$. It is now easy to see that the Hamiltonian (8.1) is a perfect square in two different ways : both operators

$$
Q=\frac{1}{\sqrt{2 m}} \boldsymbol{\pi} \cdot \boldsymbol{\sigma} \quad \text { and } \quad Q^{*}=\frac{1}{\sqrt{2 m}} \boldsymbol{\pi} \times \boldsymbol{\sigma}
$$

where $\boldsymbol{\sigma}=\left(\sigma_{1}, \sigma_{2}\right)$, satisfy

$$
\{Q, Q\}=\left\{Q^{\star}, Q^{\star}\right\}=2 H
$$

Thus, for any static, purely magnetic field in the plane, $H$ is an $N=2$ supersymmetric Hamiltonian. The supercharge $Q$ is a standard object used in supersymmetric quantum

$(2)$ This is to be compared with the Galilean supersymmetry [30] for non-relativistic Chern-Simons systems, and with the $\operatorname{osp}(1 / 2)$ found by Hughes et al. in a constant magnetic field [31].

$\left({ }^{3}\right)$ The vector or cross product of two planar vectors, $\mathbf{u} \times \mathbf{v}=\epsilon_{i j} u^{i} v^{j}$, is a scalar. 
mechanics; the 'twisted' charge $Q^{\star}$ was used, e.g., [32], to describe the Landau states in a constant magnetic field [29,31].

Let us assume henceforth that $B$ is the field of a point-like magnetic vortex directed along the $z$-axis, $B=\Phi \delta(\mathbf{r})$, where $\Phi$ is the total magnetic flux $\left({ }^{4}\right)$. Inserting $A_{i}(\mathbf{r})=$ $-(\Phi / 2 \pi) \epsilon_{i j} \mathbf{r}^{j} / r^{2}$ into the Pauli Hamiltonian $H$ in (8.1), it is straightforward to check that

$$
D=t H-\frac{1}{4}\{\boldsymbol{\pi}, \mathbf{r}\} \quad \text { and } \quad K=-t^{2} H+2 t D+\frac{1}{2} m r^{2}
$$

cf. (2.3) generate, along with $H$, an $o(2,1)$ Lie algebra (2.6). The angular momentum, $J=\mathbf{r} \times \boldsymbol{\pi}$, adds to this $o(2,1)$ an extra $o(2)\left({ }^{5}\right)$.

Commuting $Q$ and $Q^{\star}$ with the expansion, $K$, yields two more generators, namely

$$
\begin{gathered}
S=i[Q, K]=\sqrt{\frac{m}{2}}\left(\mathbf{r}-\frac{\boldsymbol{\pi}}{m} t\right) \cdot \boldsymbol{\sigma}, \\
S^{\star}=i\left[Q^{\star}, K\right]=\sqrt{\frac{m}{2}}\left(\mathbf{r}-\frac{\boldsymbol{\pi}}{m} t\right) \times \boldsymbol{\sigma} .
\end{gathered}
$$

It is now straightforward to see that both sets $Q, S$ and $Q^{\star}, S^{\star}$ extend the $o(2,1) \cong$ $\operatorname{osp}(1 / 0)$ into an $\operatorname{osp}(1 / 1)$ superalgebra. These two algebras do not close yet, though : the 'mixed' anticommutators $\left\{Q, S^{\star}\right\}$ and $\left\{Q^{\star}, S\right\}$ produce a new conserved charge, viz.

$$
\left\{Q, S^{\star}\right\}=-\left\{Q^{\star}, S\right\}=J+2 \Sigma, \quad \text { where } \quad \Sigma=\frac{1}{2} \sigma_{3}
$$

But $J$ satisfies now non-trivial commutation relations with the supercharges,

$$
[J, Q]=-i Q^{\star}, \quad\left[J, Q^{\star}\right]=i Q, \quad[J, S]=-i S^{\star}, \quad\left[J, S^{\star}\right]=i S .
$$

Thus, setting

$$
Y=J+2 \Sigma=\mathbf{r} \times \boldsymbol{\pi}+\sigma_{3},
$$

$\left({ }^{4}\right)$ Our setup can be thought of as an idealization of the spinning version of the AharonovBohm experiment [33].

$\left(^{5}\right)$ The correct definition of angular momentum requires boundary conditions. 
the generators $H, D, K, Y$ and $Q, Q^{\star}, S, S^{\star}$ satisfy

$$
\begin{array}{llll}
{[Q, D]} & =\frac{i}{2} Q, & {\left[Q^{\star}, D\right]} & =\frac{i}{2} Q^{\star}, \\
{[Q, K]} & =-i S, & {\left[Q^{\star}, K\right]} & =-i S^{\star}, \\
{[Q, H]} & =0, & {\left[Q^{\star}, H\right]} & =0, \\
{[Q, Y]} & =-i Q^{\star}, & {\left[Q^{\star}, Y\right]} & =i Q, \\
{[S, D]} & =-\frac{i}{2} S, & {\left[S^{\star}, D\right]} & =-\frac{i}{2} S^{\star}, \\
{[S, K]} & =0, & {\left[S^{\star}, K\right]} & =0, \\
{[S, H]} & =i Q, & {\left[S^{\star}, H\right]} & =i Q^{\star}, \\
{[S, Y]} & =-i S^{\star}, & {\left[S^{\star}, Y\right]} & =i S, \\
\{Q, Q\} & =2 H, & \left\{Q^{\star}, Q^{\star}\right\} & =2 H, \\
\{S, S\} & =2 K, & \left\{S^{\star}, S^{\star}\right\} & =2 K, \\
\left\{Q, Q^{\star}\right\} & =0, & \left\{S, S^{\star}\right\} & =0, \\
\{Q, S\} & =-2 D, & \left\{Q^{\star}, S^{\star}\right\} & =-2 D, \\
\left\{Q, S^{\star}\right\}=Y, & \left\{Q^{\star}, S\right\} & =-Y .
\end{array}
$$

Added to the $o(2,1)$ relations, this means that our generators span the $\operatorname{osp}(1 / 2)$ superalgebra $[11,13]$. On the other hand,

$$
Z=J+\Sigma=\mathbf{r} \times \boldsymbol{\pi}+\frac{1}{2} \sigma_{3}
$$

commutes with all generators of $\operatorname{osp}(1 / 2)$, so that the full symmetry is the direct product $o s p(1 / 2) \times o(2)$, generated by

$$
\left\{\begin{aligned}
Y & =\mathbf{r} \times \boldsymbol{\pi}+\sigma_{3}, & Q & =\frac{1}{\sqrt{2 m}} \boldsymbol{\pi} \cdot \boldsymbol{\sigma}, \\
H & =\frac{1}{2 m}\left[\boldsymbol{\pi}^{2}-e B \sigma_{3}\right], & Q^{\star} & =\frac{1}{\sqrt{2 m}} \boldsymbol{\pi} \times \boldsymbol{\sigma}, \\
D & =-\frac{1}{4}\{\boldsymbol{\pi}, \mathbf{q}\}-t \frac{e B}{2 m} \sigma_{3}, & S & =\sqrt{\frac{m}{2}} \mathbf{q} \cdot \boldsymbol{\sigma}, \\
K & =\frac{1}{2} m \mathbf{q}^{2}, & S^{\star} & =\sqrt{\frac{m}{2}} \mathbf{q} \times \boldsymbol{\sigma}, \\
Z & =\mathbf{r} \times \boldsymbol{\pi}+\frac{1}{2} \sigma_{3}, & &
\end{aligned}\right.
$$

where we have put $\mathbf{q}=\mathbf{r}(\boldsymbol{\pi} / m) t$.

The supersymmetric Hamiltonian (8.1) is the square of Jackiw's [32] two-dimensional Dirac operator $\pi \times \sigma$. But the Dirac operator is supersymmetric in any even dimensional space. The energy levels are therefore non-negative; eigenstates with non-zero energy are 
doubly degenerate; the system has $\operatorname{Ent}(e \Phi-1)$ zero-modes [32, 33]. The superalgebra (8.6) allows for a complete group-theoretical solution of the Pauli equation, along the lines indicated by D'Hoker and Vinet $[11,13]$.

Notice that the two-dimensional Dirac operator $\pi \times \sigma$ of Ref. [32] - essentially our $Q^{\star}$ - is associated with the unusual choice of the two-dimensional 'Dirac' (i.e. Pauli) matrices $\gamma_{1}^{\star}=-\sigma_{2}, \gamma_{2}^{\star}=\sigma_{1}$. Our helicity operator, $Q$, is again a 'Dirac operator' - but one associated with the standard choice $\gamma_{1}=\sigma_{1}, \gamma_{2}=\sigma_{2}$.

Acknowledgements. This review is based on joint research with L. Fehér, B. Cordani, L. O'Raifeartaigh, F. Bloore, C. Duval, G. Gibbons and A. Comtet, to whom I express my indebtedness.

\section{References}

[1] B. Cordani, The Kepler problem. Birkhäuser (2003). The o(4,2) symmetry was first found by H. Kleinert, Colorado Lecture (1966) (unpublished); A.O. Barut and H. Kleinert, Phys. Rev. 156 1541, (1967); G. Györgyi, Il Nuovo Cimento A53, 717 (1968)

[2] For a review on monopoles see, e.g., P. Goddard and D. Olive, Rep. Prog. Phys. 41, 1357 (1978)

[3] Golo, JETP Lett. 35, 535 (1982).

[4] L. Gy. Fehér Journ. Math. Phys. 28, 234 (1987).

[5] D. G. Boulware, L. S. Brown, R. N. Cahn, S. D. Ellis, C. Lee, Phys. Rev. D14, 2708 (1976).

[6] R. Jackiw, Ann. Phys. (N.Y.) 129, 183 (1980).

[7] R. Jackiw, Phys. Today 25, 23 (1972); U. Niederer, Helv. Phys. Acta 45, 802 (1972);

C. R. Hagen, Phys. Rev. D5, 377 (1972); C. Duval Thèse de Doctorat d'Etat, Marseille (1982) (unpublished).

[8] C. Duval, G. Burdet, H. P. Künzle, M. Perrin, Phys. Rev. D31, 1841 (1985); C. Duval, G. W. Gibbons and P. A. Horváthy, Phys. Rev. D43, 3907 (1991).

[9] H. V. McIntosh and A. Cisneros, Journ. Math. Phys. 11, 896 (1970); D. Zwanziger, Phys. Rev. 176, 1480 (1968); J. Schönfeld, Journ. Math. Phys. 21, 2528 (1971); L. Gy. Fehér, Journ. Phys. A19, 1259 (1986); and in Non-Perturbative Methods in Quantum Field Theory, Proc. 1986'Siófok Conference, Horváth, Palla, Patkós (eds), Singapore: World Sci. (1987). For the scattering, see L. Gy. Fehér and P. A. Horváthy, Mod. Phys. Lett. A3, 1451 (1988).

[10] A. O. Barut and G. L. Bornzin, Journ. Math. Phys. 4, 141 (1971); B. Cordani, L. G. Fehér and P. A. Horváthy, Journ. Math. Phys. 31, 202 (1990). 
[11] E. D'Hoker and L. Vinet, Phys. Lett. 137B, 72, (1984).

[12] J. P. Gauntlett, J. Gomis and P. K. Townsend, Phys. Lett. 248B, 288 (1990); C. Duval and P. A. Horváthy, Journ. Math. Phys. 35, 2516 (1994) hep-th/0508079.

[13] E. D’Hoker and L. Vinet, Comm. Math. Phys. 97, 391-427 (1985).

[14] E. D'Hoker and L. Vinet, in Field Theory, Quantum Gravity and Strings, Meudon Paris seminars 85/86, Springer Lecture Notes in Physics 280, p. 156; Lett. Math. Phys. 12, 71 (1986). E. D'Hoker, V. A. Kostelecky and L. Vinet, in Dynamical Groups and Spectrum Generating Algebras, p. 339-367, World Scientific, Singapore (1988).

[15] E. Mottola, Phys. Lett. 79B, 242 (1979).

[16] E. J. Weinberg, Phys. Rev. D20, 936 (1979).

[17] E. D'Hoker and L. Vinet, Phys. Rev. Lett. 55, 1043 (1986).

[18] L. Gy. Fehér, P. A. Horváthy and L. O’Raifeartaigh, Int. Journ. Mod. Phys. A4, 5277 (1989); Phys. Rev. D40, 666 (1989).

[19] F. Bloore and P. A. Horváthy, Journ. Math. Phys. 33, 1869 (1992).

[20] L. C. Biedenharn, Phys. Rev. 126, 845 (1962); M. Berrondo and H. V. McIntosh, Journ. Math. Phys. 11, 125 (1970).

[21] T. T. Wu and C. N. Yang, in Properties of Matter under Unusual Conditions, H. Mark and S. Fernbach (eds), Interscience, (1969).

[22] A. O. Barut and G. L. Bornzin, Phys. Rev. D7, 3018 (1973).

[23] P. A. Horváthy, Mod. Phys. Lett. A6, 3613 (1991).

[24] Gross and M. Perry, Nucl. Phys. B226, 29 (1983); R. Sorkin, Phys. Rev. Lett. 51, 87 (1983).

[25] N. Manton and G. W. Gibbons, Nucl. Phys. 274, 183 (1986); L. Gy. Fehér and P. A. Horváthy, Phys. Lett. 183B, 182 (1987); B. Cordani, L. Gy. Fehér and P. A. Horváthy, Phys. Lett. 201, 481 (1988).

[26] Z. F. Ezawa and A. Iwazaki, Phys. Lett. 138B, 81 (1984); M. B. Paranjape and G. W. Semenoff, Phys. Rev. D31 (1985) 1324. Later developments include M. Visinescu, Phys. Lett. B339, (1994); J. W. van Holten, Phys. Lett. B342, 47 (1995), A. Comtet and P. A. Horváthy, Phys. Lett. B349, 49 (1995), etc. 
[27] R. Jackiw, Ann. Phys. (N.Y.) 201, 83 (1990).

[28] C. J. Parks, Nucl. Phys. B367, 99 (1992); J.-G. Demers, Mod. Phys. Lett. 8, 827 (1993); C. Duval and P. A. Horváthy, Tours Preprint N. 60/93 (1993) (unpublished).

[29] E. Witten, Nucl. Phys. B185, 513 (1981); P. Salomonson and J.W. Van Holten, Nucl. Phys. B169, 509 (1982); M. De Crombrugghe and V. Rittenberg, Ann. Phys. (N.Y.) 151, 99 (1983).

[30] M. Leblanc, G. Lozano and H. Min, Ann. Phys. (N.Y.) 219, 328 (1992); C. Duval and P.A. Horváthy, in Ref. [12]. The bosonic galilean symmetry was pointed out by R. Jackiw and S.-Y. Pi, Phys. Rev. D 42, 3500 (1990).

[31] R. J. Hughes, V. A. Kostelecký and M. M. Nieto, Phys. Rev. D34, 1100 (1986).

[32] R. Jackiw, Phys. Rev. D29, 2375 (1984).

[33] C. R. Hagen, Phys. Rev. Lett. 64, 503 (1990); R. Musto, L. O'Raifeartaigh and A. Wipf, Phys. Lett. B 175, 433 (1986); P. Forgács, L. O'Raifeartaigh and A. Wipf, Nucl. Phys. B 293, 559 (1987). 